\author{
Marquette University \\ e-Publications@Marquette
}

2015

\title{
When Auditory and Visual Signal Processing Conflict: Cross- Modal Interference in Extended Work Periods
}

Stephen J. Guastello

Marquette University, stephen.guastello@marquette.edu

Katherine Reiter

Marquette University

Matthew Malon

Marquette University

Anton Shircel

Marquette University

Follow this and additional works at: https://epublications.marquette.edu/psych_fac

Part of the Psychology Commons

\section{Recommended Citation}

Guastello, Stephen J.; Reiter, Katherine; Malon, Matthew; and Shircel, Anton, "When Auditory and Visual Signal Processing Conflict: Cross-Modal Interference in Extended Work Periods" (2015). Psychology Faculty Research and Publications. 218.

https://epublications.marquette.edu/psych_fac/218 
Marquette University

e-Publications@Marquette

\section{Department of Psychology Faculty Research and Publications/College of Arts and Sciences}

This paper is NOT THE PUBLISHED VERSION.

Access the published version at the link in the citation below.

Theoretical Issues in Ergonomics Science, Vol. 16, No. 3 (2015): 232-254. DOI. This article is (C) Taylor \& Francis and permission has been granted for this version to appear in e-Publications@Marquette. Taylor \& Francis does not grant permission for this article to be further copied/distributed or hosted elsewhere without the express permission from Taylor \& Francis.

\section{When auditory and visual signal processing conflict: cross-modal interference in extended work periods}

Stephen J. Guastello

Department of Psychology, Marquette University, Milwaukee, WI, USA

Katherine Reiter

Department of Psychology, Marquette University, Milwaukee, WI, USA

Matthew Malon

Department of Psychology, Mount Mary University, Milwaukee, WI, USA

Anton Shircel

Kohler Corporation, Sheboygan, WI, USA 


\begin{abstract}
Auditory and visual stimuli presented at intervals of about $300 \mathrm{~m} \mathrm{sec}$ often produce miss errors in one or the other channel, which result from a bottleneck in a neural circuit associated with executive memory. The present study examined the possibility that cross-modal interference could carry over to performance units that transpire over 3 min or longer. An N-back task performed by 113 undergraduates with simultaneous auditory and visual stimuli was organised into 1-min blocks of 20 trials in 2-back and 3-back conditions. Results showed that impairment of visual processing was more frequent than impairment of auditory processing under conditions of fatigue. A substantial number of person blocks showed no such interference, however. Bottlenecks during early stages of processing may have more extensive effects on later processing than previously recognised. Further research should consider perceptual cycling in longer term tasks involving complex stimuli.
\end{abstract}

\title{
Keywords:
}

Attention, resource theory, schema theory, auditory displays, visual displays

\section{Introduction}

According to resource theory (Wickens 2002, 2008), two tasks are less likely to conflict and produce a bottleneck when performed simultaneously if the two tasks require different resources at the perception, processing, and motor responses phases of cognitive activity. A combination of an auditory and a visual task would be a prototype example of a pair of tasks that would be relatively unlikely to conflict. The principle and prototype example would be consistent with the theory of working memory (Baddeley 2003), which specifies an executive function and mental workspace function; the latter is divided between audio and visual channels, known as the phonological loop and the visuospatial sketchpad, respectively, and the other mental workspace areas that can be recruited by the executive function for use as needed. In contrast to what one would expect from resource theory, experimental conditions are coming to the foreground whereby auditory and visual signals arriving simultaneously can have inhibitory effects on each other. The events, known as attentional blink, have been observed at the levels of responses to single-mode stimuli presented in rapid succession ( $300 \mathrm{~m} \mathrm{sec}$ ), responses to dual-mode stimuli presented in rapid succession, and neural activation (Dux and Marois 2009; Haroush, Deouell, and Hochstein 2011; Kelly and Dux 2011; Marti, Sigman, and Dehaene 2012; Raymond, Shapiro, and Arnell 1992; Theeuwes et al. 2007). The typical blink experiment involves a stimulus recognition task and thus does not appear to place a high demand on working memory.

The objective of the present study is to determine the extent to which the cross-modal interference that is associated with attentional blink is visible in larger aggregates of task performance than the simple stimulus and response. Further study of the interference phenomenon could inform the design and use of person--machine systems where auditory and visual signals are (1) streaming simultaneously; (2) used redundantly to signal an event, e.g. in a vigilance task; or (3) used conjointly to identify stimuli that meet two different criteria for a single response. The present experiment is an example of (1), with the new objective of examining whether the interference phenomenon carries over to phases of cognition that transpire beyond than the first half-second after stimulus onset and 
persist for several minutes afterwards. The pertinent theoretical issues are developed next in four segments:

1. Cross-modal blink, similar phenomena, and the possible relationship of the effects to fatigue are described. One recent study is examined that indicates that cross-modal blink and interference in longer task cycles may very well be connected.

2. The possible role of cross-modal interference in $\mathrm{N}$-back tasks, which places a higher demand on memory than the standard experimental design and also requires longer time intervals between the initial stimuli and responses. An $\mathrm{N}$-back task was used in the experiment presented here. Note that there is no inherent connection between the blink phenomenon and $\mathrm{N}$-back tasks. Rather, $\mathrm{N}$-back tasks require the perceptual recognition of stimuli which is followed by further cognitive operations within working memory. It was possible to fashion an $\mathrm{N}$-back task for this experiment; however, that required attention to both an auditory and visual stimulus stream simultaneously. The time allowed to respond to the bimodal stimuli was $3 \mathrm{sec}$, which was well outside the $300 \mathrm{~m}$ sec or less in which the cross-modal attentional blink effect occurs.

3. The premise of the experiment is that cross-modal interference can persist for repeated stimuli that transpire over 1-3 min or longer. There are two parts to the explanation for why such persistence could occur, based on the current state of science. First, perceptual cycle theory (Neisser 1976) is introduced as a plausible explanation for how the cross-modal inhibition that occurs in blink studies could persist in cognitive activities that transpire over longer time horizons. Second, some recent work in neurocognitive dynamics supports both perceptual cycling theory and explains the longer term impact of interference further. The neurological mechanisms engage a cycling pattern of their own that consists of activation and inhibition. That type of cycling pattern appears to explain the functioning of the bottleneck circuit that was recently discovered as the essential cause of the basic attentional blink phenomenon.

4. The fourth step integrates the foregoing themes with broader issues concerning bottlenecks and fatigue.

\section{Some connected cognitive processes}

\subsection{Blink and related phenomena}

The blocking effect (Bills 1931) was observed when an operator viewed a stream of discrete stimuli over an extended period of time and gave a response. When fatigue sets in the operator could miss a stimulus entirely; response time would go to infinity for that trial and then return to the normal range for the subsequent trial. The phenomenon has been looking for an explanation in cognitive fatigue research ever since (Ackerman 2011). The discontinuity in performance during blocking was interpreted as one of several examples of catastrophic shifts in performance under conditions of fatigue (Guastello and McGee 1987). Catastrophic shifts imply two control parameters; one control parameter would be the amount of work done during the time on task, and the other would be an ability that compensated for the fatigue induced in the resource that was primary to the task (Guastello 2003; Guastello, Boeh, Gorin et al. 2013; Guastello, Boeh, Schimmels et al. 2012; Guastello, 
Boeh, Shumaker, and Schimmels 2012; Guastello, Malon et al. 2014; Guastello et al. in press; Guastello, Shircel et al. 2013). When the operator assumes greater workload during a given period of time, greater fatigue can be expected; a functional relationship between time on task, workload, and fatigue has been recently identified (Ahmed et al. 2014). Bills' blocking effect, however, has not been explained much further since it was first reported.

The attentional blink is one of the two similar phenomena that have been observed during the first half-second of the presentation of the signals or stimuli; the companion phenomenon is the interstimulus refractory period (IRP). Like the single neuron that cannot fire twice in response to two stimuli that arrive too-close together in time, neural circuits in the prefrontal gyrus appear to have the same limitation in re-orienting to a stimulus appearing at an interval less than one half-second. IRPs are more evident when either auditory or visual stimuli appear in rapid succession. When interstimulus intervals exceed the IRPs, which are less than $300 \mathrm{~m} \mathrm{sec}$, however, an attentional blink with either the auditory, visual, or cross-modal audiovisual stimuli can occur (Marti et al. 2012).

Not all experimental participants exhibit attentional blink under the same experimental conditions, leading some researchers to study individual differences (Kelly and Dux 2011; MacLean and Arnell 2010; Van Dam, Earleywine, and Alterriba 2012). If the participant does not miss the stimulus altogether, there is still a slightly extended response time to one or the other stimulus. When crossmodal blink does occur, however, auditory stimuli interrupt visuals more often than vice versa (Haroush et al. 2011; Marti et al. 2012). The alleged source of conflict between stimuli is that, although the auditory and visual circuits start in separate places, they both bottleneck through a common circuit that is associated with the executive functions of working memory (Figure 1). The circuits that are responsible for IRPs are thought to be the same as those responsible for attentional blink. The circuits separate again as later processing continues.

Figure 1. Schematic of the neural system that produces IRP, cross-modal blink, and possibly interference at later stages of processing.

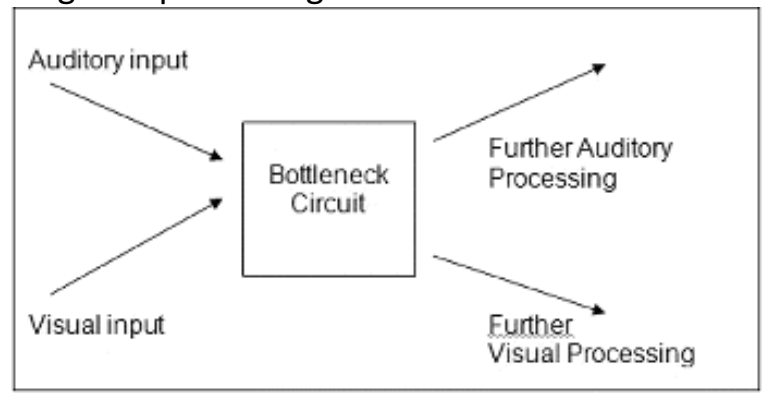

In an ergonomics application, Mkrtchyan et al. (2012) attempted to induce periodic attention to a display for a vigilance task by adding auditory cues. In one experimental condition, the display was equipped with an auditory alert that signalled events on the screen that required responses such as destroying an enemy target; no auditory alerts were produced in the second condition. Target events were planned to occur in a cyclic fashion over time. The study was intended to determine whether the auditory alerts would improve performance overall and whether they would exaggerate cyclic attention to the task compared to distraction activities. Participants were permitted to engage in 
distractions while working, such as doing tasks on their laptops, or using cell phones for data but not actual phone calls; snacks and soft drinks were also provided as distractions.

The results showed that the performance was actually better without the auditory alert, which was inconsistent with the time-honoured principle of redundancy enhancing reliability. Participants were also classified as directed in their attention to the task, divided in attention, or distracted based on an analysis of videotapes of their behaviour while working on the task. The auditory alerts did not improve attention to the task, except in the case of one out of the nine participants who was the most distracted. Although Mkrtchyan et al.'s study did not specifically address attentional blink or subsequent interference, either one could have been responsible for the conflict they observed when auditory cues were added to the visual cues.

\subsection{Attentional blink in N-back tasks}

Of the several hundred studies on attentional blink, we are aware of only one attempt (Wynna et al. 2006) to explore the connection between the events of the first half-second and events in subsequent processing in working memory that could be captured in an $N$-back experimental task. In an $\mathrm{N}$-back task, the research participants attend to a series of auditory or visual stimuli and need to remember whether they saw a particular stimulus just previously (1-back), two events previously (2back), three events ago (3-back), etc. $N$-back tasks are thought to place a strong demand on the executive function component of working memory (Kane and Engle 2002), particularly the updating function. Performance on $N$-back tasks would thus be susceptible to fatigue effects (Logie 2011), which are usually observed as performance decrements over time (Ackerman 2011; Guastello et al. in press; Guastello Boeh, Shumaker, and Schimmels 2012; Guastello, Boeh et al. 2012, 2013; Guastello, Malon et al. 2014; Guastello and McGee 1987).

Our search of the experimental literature indicated that previous studies that used $N$-back tasks for different purposes seldom used tasks more difficult than 2-back. The present experiment involved tasks in 2-back and 3-back modes with auditory and visual stimuli presenting simultaneously. Importantly, $\mathrm{N}$-back tasks involve memory processes that extend beyond the first $500 \mathrm{~m} \mathrm{sec}$. Associations between current and remembered stimuli could involve processes extending up to $9 \mathrm{sec}$ in the 3-back task used here. Furthermore, the present study investigated whether cross-modal interference can occur at behavioural aggregates of $60 \mathrm{sec}$ (20 stimulus--response units). If interference occurs at larger aggregates, it could have some useful implications for design of interfaces that involve auditory and visual stimuli.

The concern with aggregation stems from the principle of time ecologies (Koehler 2003). Many dynamic events result from processes at different time scales (Guastello 2010; Koehler 2003; Mandelbrot 1997; Zaheer, Albert, and Zaheer 1999), or come into better focus when a large enough time scale is adopted (Guastello, Peressini, and Bond 2011). Patterns in neural activity, intention, memory, and those of machine systems all combine to produce holistic time series of system performance. The combinational structure can result from the synchronisation of cognitive and other processes, or from other emergent processes in person--system interaction, and there is no a priori reason to assume that the resulting pattern is faster, slower, or the average of contributing temporal patterns. Although there are limits to the number of temporal dynamics that can be assessed in one experimental study, the concept of time ecologies is compelling enough to investigate the possibility 
that aggregates of stimulus--response patterns that are larger than the single trials in a standard blink study can exist and be informative. In the particular case of $\mathrm{N}$-back tasks, the task itself requires memory of stimuli over two or three trials, and is thus likely to induce a level of patterning. Extended time working on such tasks is plausibly embedded within a broader temporal dynamics associated with cognitive workload and fatigue.

\subsection{Perceptual cycling}

Perceptual cycle theory (Neisser 1976) offers some plausible descriptions and explanations of events that occur beyond the first $500 \mathrm{~m} \mathrm{sec}$, form aggregations, and cycle in their behaviour, particularly when auditory--visual inhibition occurs. The events appear to be self-organising from the bottom-up and top-down.

\subsubsection{Cognitive principles}

A common feature of perceptual cycle theory and ecological psychology (Gibson 1979) is that people are actively looking for affordances. Attention to one target in the environment diverts attention from other potential targets. Perception and attention are essentially the same process, according to Neisser (1976), who proposed, furthermore, that there is no need to assume that there is a separate cognitive filter operating. Divided attention and distracted attention were sufficient explanations for incomplete or degraded perception-action outcomes.

The perceptual cycle is a sequence of anticipation, explorations, and information pickup. If the perceived target connects to something meaningful to the active search, the response ensues. The ability to recognise and respond to affordances requires schemata, which are mental structures of perceptual elements that are essentially template recognition processes. The perception-action combination is also learned and consolidated and completes the cycle. The perception-action sequences are the core elements of recognition-primed decision-making (Klein 1989).

Schemata involve anticipation of what to expect and do next, and further connections between perceptual cycles and situation awareness have been made (Stanton, Chamber, and Piggott 2001). A possible negative consequence of the cycle is that incorrect schemata can be activated, at which time the incorrect action sequence proceeds like the proverbial freight train rolling downhill (Plant and Stanton 2012). The amount of time it takes to find and execute a schema can be substantial (Spivey and Dale 2006); it would depend on its rule complexity, and thus its demand on working memory, and the ambiguity of the perceptual target. The availability of an existing schema could pre-empt the development of a new schema when needed, although new schemata can eventually differentiate given enough experience.

In an illustrative experiment, Ye, Cardwell, and Mark (2009) showed the experimental participants a variety of objects, each of which had one or two affordances that were not part of the original design intention of the object. An example would be the use of a drinking glass that could be used to dig sand or pound a cracker or other object. The participants were able to find one affordance, but often failed to find two when the two were present. Once one schema was activated it was not readily changed. Dual affordances were more difficult to detect if the affordances required grasping the object in different ways; in other words, the action portion of the schemata was different. 
The key points of Ye et al.'s experiments were, for the present purposes, are as follows. (1) Perceptual cycles are relatively intact. (2) One schema needs to be executed before a different one (for another affordance) can be engaged. (3) The schematic inhibition (or interference) bears some resemblance with cross-modal attentional blink, but on a longer time horizon. Although there might not be a cognitive filter operating on the perception of affordances, the circuit that is responsible for the bottleneck in blink could be responsible for limiting the number of schemata that can be executed at once.

\subsubsection{Neurocognitive cycling}

The onset of the stimuli that trigger the schemata is marked by the onset of gamma-band electroencephalogram (EEG) activity that is produced by excitatory and inhibitory oscillations in neural activity (Baird 1999; Bressler and Freeman 1980; Ward 2004; Whittle 2010). The gamma activity observed in the blink process is independent of the P300 wave activity that is commonly associated with workload events, according to Batterink, Karns, and Neville (2012). When multiple schemata are engaged by the flow of stimuli, oscillation functions coordinate and synchronise (Ward 2004; Whittle 2010). Furthermore, there is some concern that the cyclical timing of stimuli in psychology experiments also induces oscillations of cognitive functions that synchronise with the natural ones. Dynamically, the experimental medium induces a strong oscillator, whereas the natural world is more likely to offer stimuli on a chaotic basis (Gregson 1992; Hancock 2013). Forcing oscillation is, incidentally, one of the several effective means for controlling chaos (Guastello 2006; Ott, Grebogi, and Yorke 1990). The level of synchronisation between neural structures is relatively modest, however, compared to the rigid entrainment that can be produced in mathematical experiments (Whittle 2010). The level of synchronisation depends, at least in part, on the similarity of the schemata and whether they generate equal activity levels in the cortex at the same time (Baird 1999). The distinctiveness of one stimulus compared to another appearing in rapid succession would be one source of desynchronisation produced by different activity levels (Schadow et al. 2007).

The principle of minimum entropy (Hong 2010; Guastello, Gorin et al. 2013; Guastello, Reiter, et al. 2014) or free energy (Friston 2010) appears to be operating here. Neural aggregates gravitate toward the greatest efficiency by finding and using the smallest number of degrees of freedom that accomplishes the goal sufficiently. By the same token, a certain amount of variability needs to remain to respond properly to the range of stimuli and situations that could be encountered. Thus, the reduction of degrees of freedom, which occurs with practice, serves to reduce the number or complexity of schemata. The accommodation of distinctive stimuli, however, would raise the number of degrees of freedom or the complexity of schemata.

It also appears that learning processes can shape both the synchronisation of schemata and the schemata themselves, resulting in an interesting emergent process. Some blink studies used visual stimuli in which the targets had two distinctive features, but the two features appeared in stimuli that were presented close together in time within the attentional blink window. After a sufficient number of trials was presented in that fashion, the two target features were perceived as an iconic whole containing both features, and the experimental participants had difficulty reporting which of the two target features appeared first (Akyurek et al. 2012). Thus, another example of self-organisation occurs when the two features merge into an intact object. Thus, the participants could perceive the two 
target features from the same mode but could not process them separately. Simultaneous auditory and visual stimuli have not been reported to form a similar gestalt, however. Rather, the opposite appears to be true in the experiments on cross-modal blink. Thus, a different process is probably underlying the cross-modal effects, which could be closer to the gamma desynchronisation induced by distinctive stimuli during attention cycling.

It is now possible to assemble the foregoing ideas into a plausible explanation for interference phenomena. Because activation-inhibition cycles are pervasive in neural architecture, the cycle is probably the core mechanism behind the functioning of the bottleneck circuit that starts the perceptual phase of the attentional blink process, allowing either auditory or visual information to pass through while inhibiting the other. Again, not everyone is susceptible to cross-modal blink, so there are instances where both types of stimuli pass through correctly, which also means that working memory could process both types of stimuli. As mentioned earlier, the circuit that is responsible for blink could likely be responsible for the number of schemata executed at the same time. If a learning process is involved, the schemata for anticipating stimuli and responding to them would be differentially developed. The differential development would in turn be responsible for other interference effects on performance observed over extended periods of time.

A connection between interference and fatigue was made earlier. Because fatigue and practice effects are occurring simultaneously at different rates and pushing performance in different directions, the effect of interference would probably not be clearly visible until after a phase of learning (of schemata) has completed. Underneath the performance decline is a reduction in the degrees of freedom used to perform and an increase in variability in performance. At this point, the individual stops performing the task or redistributes degrees of freedom in an advantageous manner (Hong 2010). Redistribution can take different forms depending on what the task allows, but one manifestation is akin to a secondwind effect when performance improves again (Guastello, Boeh et al. 2013). For the present purposes, it would be advisable to study interference phenomena during an epoch of fatigue rather than an epoch of learning or redistribution.

\subsection{The location of bottlenecks}

Efforts to uncover and locate the presence of bottlenecks have been an integral part of the research on cognitive channel capacity since the beginning of those investigations (Kantowitz 1985; Pashler and Johnston 1998; Welford 1952, 1980). One such bottleneck was identified in the earliest processing stage by manipulating stimulus onset asynchrony, which is the common denominator in IRP and crossmodel blink. When the presentation times of two stimuli are too close together, the response time to the second stimulus is impaired, but as the asynchrony becomes greater, the impairment reduces to an asymptotic minimum (Pashler and Johnston 1998).

The general rule took shape that stimuli that relied on different resources lessen the odds of incurring a bottleneck, but limits to channel capacity were still apparent, nonetheless. The quantification of channel capacity remains elusive, however, and workload measurements are at best relative to the task combinations being investigated (Guastello 2014, 115).

There is also evidence to support the existence of bottlenecks in the middle stages of cognitive processing that are independent of those found during the first $300 \mathrm{~m} \mathrm{sec}$ of stimulus onset (Johnson, 
McCann and Remington 1995). Cognitive strategies such as chunking input for 'batch processing' can also help avoid bottlenecks during the processing phase. A batch from one task proceeds through the central process before the batch from the second task (Pashler and Johnston 1998). If the stimulus flow is relatively unpredictable, batching can reduce the number of task switches required in a given amount of time and thus reduce fatigue (Guastello, Gorin et al. 2012). Batching and buffering would denote attention cycling at an intermediate stage of processing if it did not already occur at the initial stage.

Although brain circuitry involves massively parallel processing, the bottlenecks themselves are pockets of serial processing (Marti et al. 2012; Pashler and Johnston 1998). The neural structures thought to be responsible for the bottlenecks have only begun to be identifiable through brain imaging technologies. Marti et al. (2012) found a bottleneck circuit in an area of the prefrontal cortex that is also associated with decision-making. That particular finding strongly suggests the involvement of the executive functions of working memory, which in turn suggests that cross-modal interference could be more pronounced under conditions of fatigue. Furthermore, even if the stimulus stream consisted of a single mode (e.g. visual) and the IRPs were not excessively short, the stimuli would still be routed through the critical circuit which, if fatigued, could produce the blocking effect initially reported by Bills (1931).

Because brain circuitry involves massively parallel processing, however, it is possible that individual differences could explain whether a particular bottleneck could occur for some people and not others, and whether specific combination stimulus or task features are always responsible for triggering the bottleneck. Such differences could explain the reports that cross-modal blink does not happen to all experimental subjects, as mentioned earlier. Individual differences that capture an individual's elasticity versus rigidity of response to changing workloads in broader circumstances are currently under investigation (Guastello, Shircel, Malon, and Timm 2015; MacLean and Arnell 2010).

\subsection{Hypotheses}

The $N$-back task used in this experiment presented auditory and visual stimuli simultaneously. The two primary hypotheses for this study were as follows. (1) Cross-modal interference could be detected at aggregation levels of 20 trials of an $\mathrm{N}$-back task, which constituted approximately one min in real time. (2) Consistent with previous results (Haroush et al. 2011; Marti et al. 2012), auditory detection or processing should interfere with visual processing more often than vice versa. More specifically, we expected that the extended sequences of visual errors would be more prevalent than the extended series of auditory errors.

The analysis of the present experimental data was designed to allow for the possibility that crossmodal interference did not happen to all participants, which was also reported in previous studies. Also, because fatigue was implicated in the blocking effect, which bears some resemblance to blink, a trend analysis of performance over 15 blocks of 20 trials was conducted to ascertain the point where the amount of time on task required for learning the task ended and the onset of fatigue began. A pilot study (not reported here) suggested that a substantial performance decrement occurred at the eighth block and was followed by performance improvements. It was important for this study not to conflate errors associated with incomplete learning with those that could result from interference in working memory. 
One group of analyses was based on all participants' responses to six blocks of trials pooled together. A second group of analyses examined within-person differences in auditory versus visual errors over the six blocks of trials. In both cases, if decrements in visual and auditory performance were proportional to each other (in the sense of $r$ ) or more consistent than inconsistent (in the sense of $\chi^{2}$ ), the results would favour an interpretation of the performance decrements arising from cognitive workload or fatigue. If there were a negative correlation between auditory and visual performance, or inconsistent performance deficits in both modalities, an inhibitory or interference effect was in operation. Also in both cases, the previous research indicated the possibility that the negative or inhibitory effects would only be evident after the people who showed no cross-modal effect in either modality were excluded from the analysis, which would signify the presence of a moderating effect. The origins of the moderating effect have not been identified yet, but the extant literature indicates that it exists.

\section{Method}

Participants were 113 undergraduates from a Midwestern University. Of that number 34 were male, and 79 were female. Their mean age was 19.35 years; $96.4 \%$ were between the age of 18 and 22 .

The $N$-back task was a configuration that was available from the Brain Workshop interactive web site (Hoskinson and Toomin 2012). Participants were seated in front of a desktop computer with a 33-cm diagonal monitor. The stimuli consisted of an auditory and a visual element. The auditory element was a spoken letter of the alphabet. There were two possible visual stimuli, depending on the random assignment of participants to experimental conditions. Participants in one condition viewed stimuli consisting of six solid geometric figures (semi-circle, square, triangle, pentagon, five-pointed star, and hexagon). In the second condition, they viewed stimuli consisting of 24 cartoon faces, an example of which appears in Figure 2. The visual stimuli were presented in blue in the centre of the display against a white background. The comparison of performance between the conditions using geometric and cartoon faces was based on findings that attentional blink was less severe when the visual stimuli were faces with emotional content (deJong et al. 2009; Musch, Engel, and Schneider 2012; Van Dam et al. 2012). The available set of 24 faces was also more comparable to the number of letters in the alphabet that were used in the auditory stimuli. Stimuli were randomly chosen by the programme except when it wanted to display a correct $N$-back.

Figure 2. Example of a cartoon face stimulus.

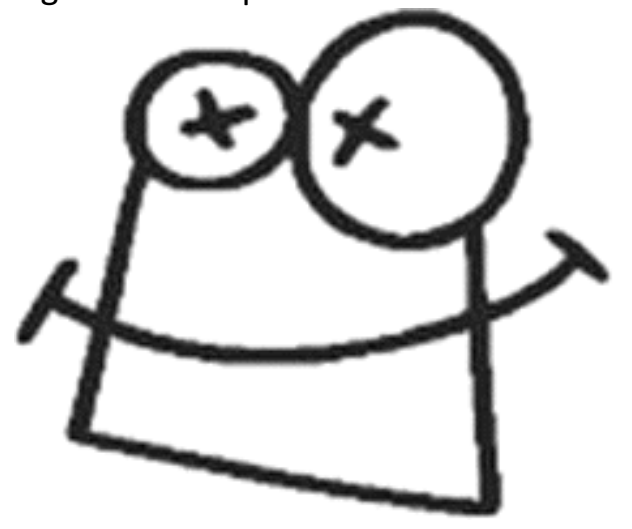


The participants completed 15 blocks of 20 trials in the 2-back mode, and 15 blocks of 20 trials in the 3back mode on a desktop computer within a controlled laboratory setting. There were no scheduled rest periods except for the time required to load the 3-back task, which required less than $1 \mathrm{~min}$. The auditory and visual stimuli were presented at 3-sec intervals from the beginning of one stimulus to the beginning of the other.

The visual stimulus appeared for $500 \mathrm{~m} \mathrm{sec}$ in the middle of the screen and then disappeared. The duration of the auditory stimuli was also $500 \mathrm{~m} \mathrm{sec}$. The instructions for which key to press remained at the bottom of the screen for the duration of the session. The system automatically recorded the accuracy rates for the audio, visual, and total performance measures. Participants received feedback about their accuracy on all three measures at the end of each block of trials.

The participants were instructed to press the $\mathrm{J}$ key if the auditory stimulus matched the $N$-back, and the $L$ key if the visual stimulus matched the $N$-back. The system could accept both key presses during the interval or simultaneously. The streams of stimuli were programmed to include $12.5 \%$ 'trick' or 'interference' trials such that auditory and visual stimuli would repeat but at the wrong $N$, either one step too soon or one step too late. The responses for the first two stimuli in 2-back and the first three stimuli in 3-back were not counted toward the accuracy rate.

The baseline accuracy rate, which could be obtained by responding 'yes' to all stimuli, was 4 per block, or $22 \%-24 \%$ depending on whether it was a 2 -back or 3-back and a small amount of random variation in the algorithm that generated stimuli. The minimum accuracy rate obtained by the participants in the blocks of trials used for the interference-related analyses was $0.0 \%$, which was obtained by $5 \%-21 \%$ of participants, with the percentage of participants depending on the block, $N$, and audio or visual nature of the stimuli.

\section{Results}

\subsection{Performance trends}

The goals of the analysis of variance (ANOVA) analyses were to determine the critical range of blocks of trials when fatigue set in and to determine whether the geometrics or cartoon faces produced any differential effect on performance. Thus, the dependent measure was the accuracy rate of performance on both auditory and visual stimuli together. The 2-back and 3-back data were analysed separately because of the large number of degrees of freedom that would be spent on possible interaction terms. The ANOVA models had one repeated effect and one fixed effect. The repeated effect was blocks of trials, 15 levels. The fixed effect was a type of visual stimuli, 2 levels.

For the 2-back task, there was a significant main effect for blocks of trials $(F(14,1554)=5.01, p<$ $\left..01, \eta_{p}{ }^{2}=.04\right)$. There were significant polynomial trends up to the fifth order $(F(1,1554)=3.56, p<$ $.10, \eta_{p}^{2}=.03$ ), meaning that there were four bends in the performance curve; Figure 3 illustrates the performance curve for auditory and visual accuracies combined. Tukey's post hoc tests (using $p<.05$ ) showed that block 1 was different from all other blocks. Block 2 was different from blocks 10, 11, 14, and 15. Block 4 was different from block 14. Blocks 5 and 7 were both different from blocks 11 and 14, and block 8 was different from block 14 . None of the other comparisons were statistically significant. There were thus two conclusions from the temporal patterns. First, block 1 was clearly a learning trial. 
Second, there were fatigue recovery dynamics occurring such that there were peaks in performance at trials 11 and 14, and troughs in performance at blocks 5, 7, and 8.

Figure 3. Mean per cent accuracy for auditory and visual stimuli combined for 15 blocks of trials for the 2-back and 3-back tasks.

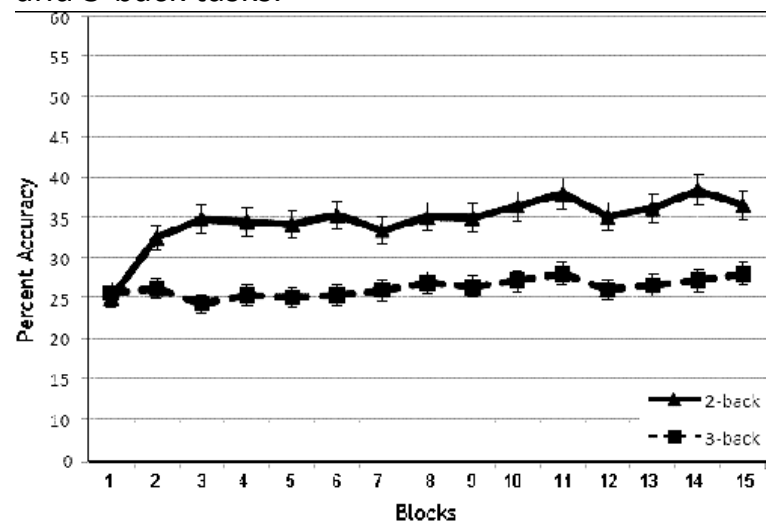

The main effect for type of visual stimulus was not significant for the 2-back task $(F(1,111)=0.00)$. The interaction between blocks and type of visual stimulus was not significant either $(F(14,1554)=0.73)$.

For the 3-back task, the main effect for blocks of trials was not significant $(F(14,1526)=1.00)$. The linear trend was significant, however, and indicated an upward trend in performance $(F(1,1526)=$ $4.13, p<.01, \eta_{p}{ }^{2}=.04$; Figure 3$)$. The other polynomial trends were not significant. An interaction between blocks and type of visual stimuli was significant, but the effect was very small $(F(14,1526)=$ 1.66. $\left.p<.10, \eta_{p}{ }^{2}=.01\right)$. Inasmuch as the interaction was probably very idiosyncratic and not readily explained, it was not pursued further.

\subsection{Interference}

Based on the results of the pilot study and the ANOVA results with the larger controlled sample, three blocks of trials were selected for analyses from the middle of the time series - blocks 7, 8, and 9 from both the 2-back and 3-back tasks. The full time series of auditory and visual performances are shown in Figure 4 for reference purposes.

Figure 4. Mean per cent accuracy for auditory and visual stimuli separately on 15 blocks of trials for the 2-back (upper) and 3-back (lower) tasks. 

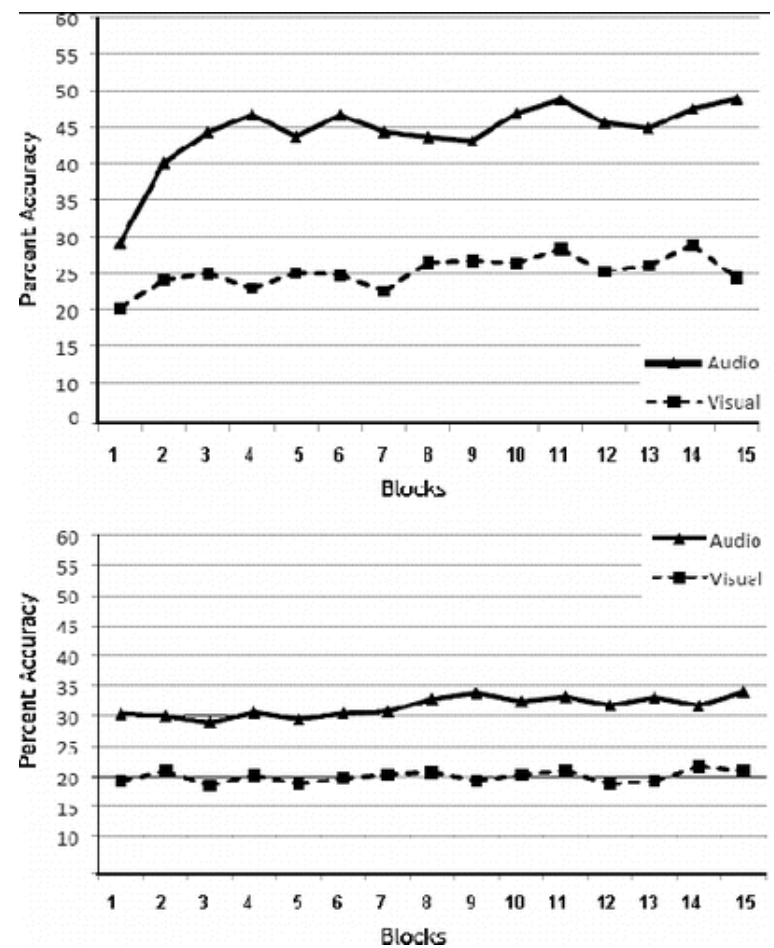

The histograms for blocks 7, 8, and 9 (Figures 5 and 6) illustrated bimodality whereby the people who performed at $0 \%$ accuracy for the entire block of trials were separating from the others. The effect was more pronounced for the visual stimuli.

Figure 5. Histograms of per cent accuracy for auditory (left column) and visual stimuli (right column) on critical blocks 7, 8, and 9 of the 2-back task.
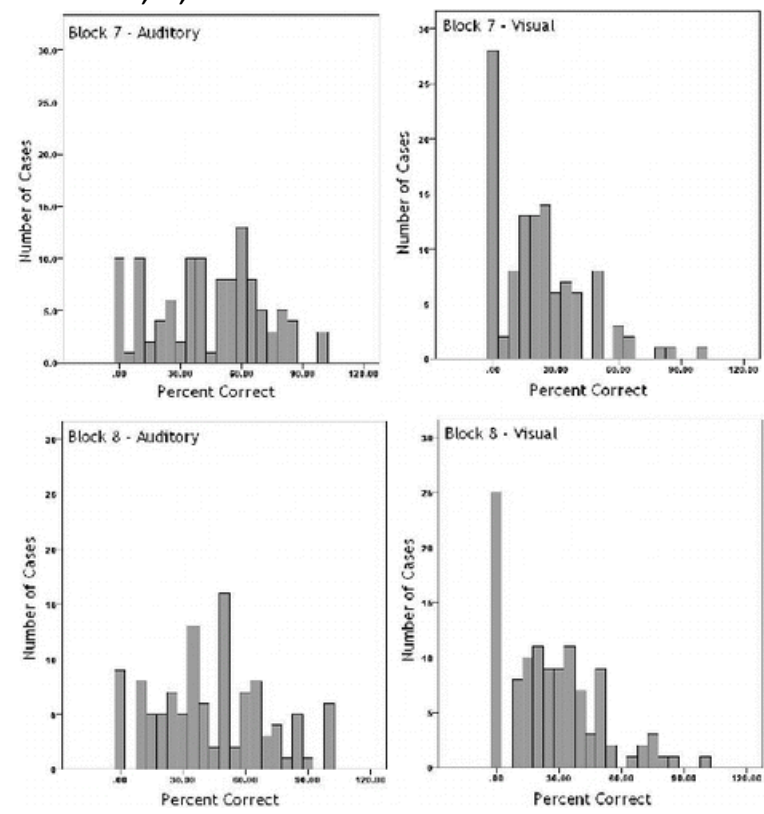

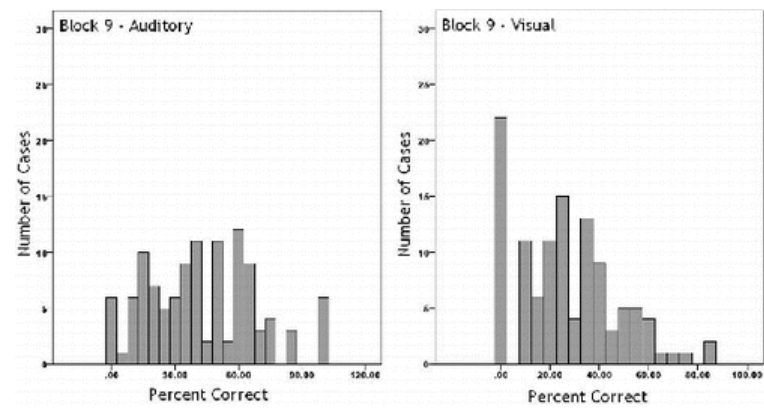

Figure 6. Histograms of per cent accuracy for auditory (left column) and visual stimuli (right column) on critical blocks 7,8 , and 9 of the 3-back task.
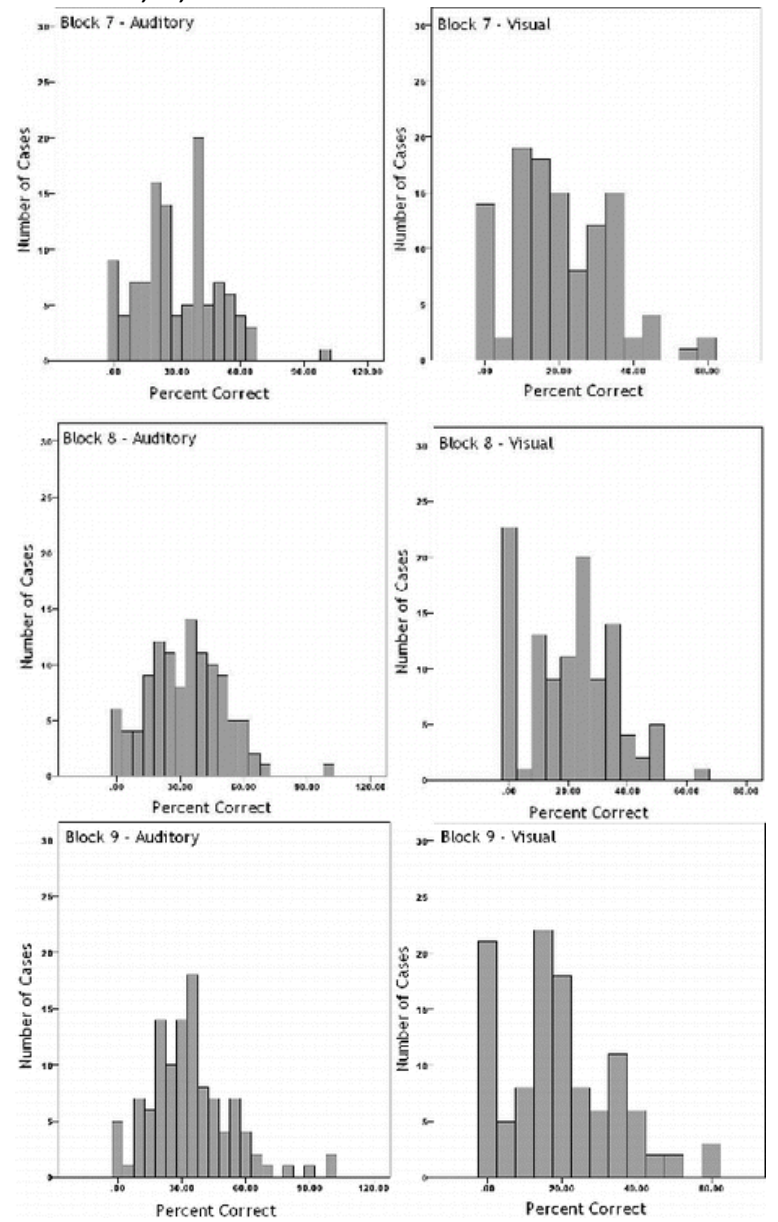

The frequencies of these auditory and visual interferences were organised for chi-square tests as follows. Data from all 113 participants in blocks 7, 8, and 9 of both the 2-back and 3-back tasks were organised into a $2 \times 2$ format (Table 1 ). Auditory and visual stimuli were dichotomised as complete errors (score $=0 \%$ ) or less than complete error (score $\geq 1 \%$ ). There were a total of 130 complete visual errors and 39 complete auditory errors.

Table 1. Number of participants with complete visual and auditory error.

\begin{tabular}{|l|l|l|l|}
\hline & Visual accuracy $\mathbf{0} \%$ & Visual accuracy $\mathbf{\geq 1 \%}$ & Row total \\
\hline Auditory & 11 & 28 & 39 \\
\hline Accuracy = 0\% & & & \\
\hline Auditory & 119 & 520 & 639 \\
\hline
\end{tabular}




\begin{tabular}{|l|l|l|l|}
\hline Accuracy $\geq 1 \%$ & & & \\
\hline Column total & 130 & 548 & 678 \\
\hline
\end{tabular}

The first test was a measure of association between complete errors on auditory and visual stimuli. A significant association would show whether complete errors were consistent on both channels or inconsistent. If they were consistent, then the higher error rates could be relegated to fatigue or other sources of human error rather than the conflict between modalities. The test was not significant, however, $\left(\chi^{2}=2.18 ; p>.05\right)$.

Given that prior experimental evidence showed that not all research participants experienced interference, the problem was reframed as two questions: if the individuals showed complete error on auditory stimuli, were they more or less likely to show complete error on visual stimuli? If the individuals showed complete error on visual stimuli, were they more or less likely to do so on auditory stimuli? The tests showed that those who showed complete error on auditory stimuli were less likely to do so on visual stimuli $\left(\chi^{2}=7.41, p<.01\right)$. Furthermore, those who showed complete error on visual stimuli were more likely not to do so on auditory stimuli $\left(\chi^{2}=109.86, p<.001\right)$. The latter effect was stronger than the former; complete visual error was more prevalent than complete auditory error, which was also consistent with previous research results.

\subsection{Within-person analyses}

The foregoing analyses were based on all participants' responses to six blocks of trials. The next analyses examined within-person differences in complete auditory versus complete visual error over the six blocks of trials. Performance scores for each person on each block were dichotomised so that a complete auditory error on a block was scored 0 and 1 otherwise. The scores were summed over the six blocks to create a variable totally wrong auditory blocks, which had a range of 0-6. The same scoring procedure was applied to visual performance data to create a variable totally wrong visual blocks.

Frequency distributions for the two totally wrong variables appear in Figure 7. Of the 113 participants, 84 did not exhibit complete errors on the auditory stimuli on any of the six blocks (score =6). Only 45, however, did not exhibit complete errors on the visual stimuli on any of the six blocks. Three participants exhibited complete errors on auditory stimuli on three or more blocks, and 13 had exhibited complete errors on visual stimuli on three on more blocks. The likelihood $\chi^{2}$ test $(42.022, \mathrm{~d} f=$ 6 , Cramer's $V=.43, p<.001$ ) indicated that the two distributions were significantly different. Thus, from the perspective of individual analysis over six blocks of trials, interference is more likely on visual stimuli than on auditory stimuli.

Figure 7. Histograms of totally wrong blocks for auditory and visual errors occurring over the six critical blocks of trials. Low scores indicate that the participant completed several blocks of trials that were completely inaccurate. 

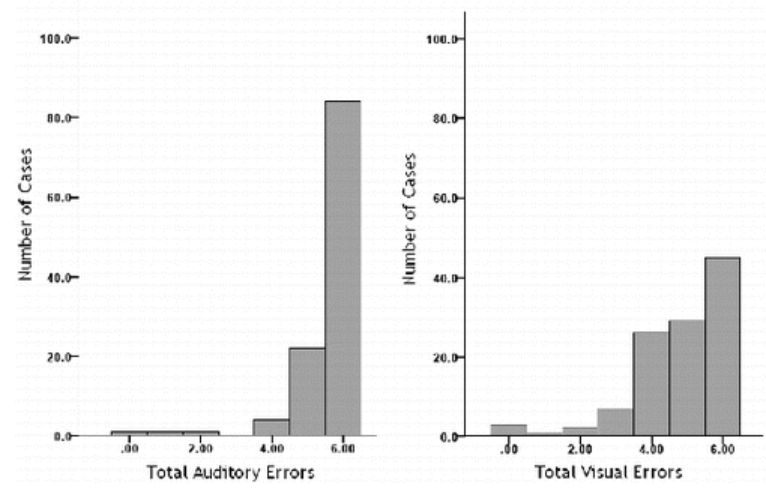

If there was an auditory-to-visual inhibition effect, there should be a negative correlation between totally wrong auditory blocks and totally wrong visual blocks. A scatterplot (Figure 8 ) showed that the join distribution was not bivariate normal. The correlation, nonetheless, was actually positive $(r=$ $.21 p<.05)$ indicating that errors in one modality were proportional to errors in the other modality. The positive correlation would suggest that the participants' errors were the result of workload demand or fatigue.

Figure 8. Scatter plot of total auditory error and total visual error over the six critical blocks of trials. Low scores indicate that the participant completed several blocks of trials that were completely inaccurate.

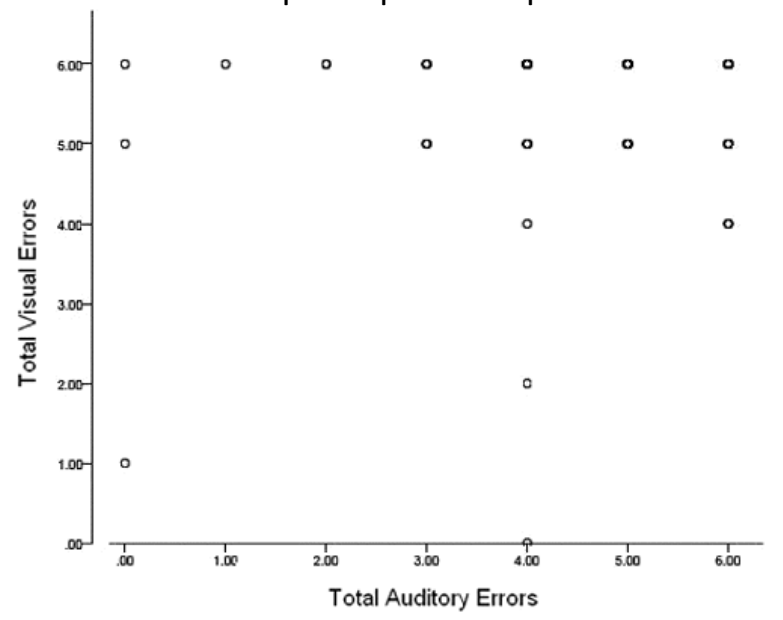

The positive correlation could have been produced, however, by the clump of 34 cases that did not produce totally wrong blocks in either mode, and the one outlier who produced totally wrong responses on five out of the six audio blocks and all six visual blocks. When those cases were dropped, the correlation based on the remaining 78 of cases dropped to .13 (NS). Although the results did not support a direct oscillation or inhibition effect, they did support the distinction between individuals who showed interference and those whose errors could arise from cognitive workload or fatigue.

\section{Discussion}

\subsection{What was learned?}

The objective of the study was to examine the possible existence of cross-modal interference in cognitive task sequences that were longer term than single stimulus events in which blink has been 
reported previously. The interference effect would have important implications for theories of cognitive workload as well as for practical application. Although cognitive fatigue was not a primary focus of the present study, recent studies now support the notion that the behavioural effects of workload, practice, fatigue, and recovery are intertwined. Thus, the experimental results reported here were predicated on situations where fatigue was likely and learning and recovery effects were less likely. It now appears that workload or fatigue effects moderate other cognitive events; this possibility needs to be explored further.

In an examination of the previous studies concerning attentional blink, it was necessary to separate the effect called 'blink' into two subtypes that were better characterised by IRP and those of the crossmodal variety; the latter were of concern here. Cross-modal blink involves some cycling between attention to auditory and visual stimuli, although the length and consistency of this particular cycle are still to be determined. Neurological evidence (Marti et al. 2012) indicates that there is a bottleneck circuit involved in both IRP and cross-modal blink and that there are other widespread oscillatory functions (Whittle 2010) that either give rise to, or are affected by cyclic attention and engagement of schemata.

Longer term tasks with dual-mode stimuli now appear susceptible to cross-modal interference, but also involve cognitive-behaviour units that engage after the critical period of $300 \mathrm{~m} \mathrm{sec}$ and cycle between one perception-action schema and another.

The results of the present study showed that cross-modal interference does occur when mixed auditory and visual stimuli are processed simultaneously and that the effect is prominent at behavioural aggregates (blocks) of 20 trials, as evidenced by the visible bimodality in most of the histograms in Figures 5 and 6 . A comparison with the histograms in Figure 7, however, indicates that individuals who produced wrong responses on a full block of trials are usually not the same ones who produced totally wrong responses on the other blocks of trials. Totally wrong responses in the visual channel were observed on the six blocks tested, however, for $11.5 \%$ of the participants for three out of six min (six blocks) of work time studied. Consistent with previous studies on attentional blink, interference on the $\mathrm{N}$-back task did not happen to all experimental participants under the same conditions. Furthermore, auditory and visual interferences both occur, but are unlikely to occur simultaneously. Auditory stimuli tend to inhibit visuals more often than vice versa.

The negative proportionality (correlation) between auditory and visual performances that would have supported a simple inhibitory effect between the two modalities within individuals was not found. If the interference effect were simply a manifestation of cognitive workload or fatigue, then decrements and peaks in performance in both modalities would be positively correlated within persons, which was roughly the case. The relationship was held together, however, by the extreme performers who did not exhibit interference in either mode for all six blocks of trials and one who did so on all six blocks in the visual mode and five out of the six blocks in the auditory mode. The pattern of results obtained here would be consistent with the interpretation that, after eliminating the contributions of fatigue, attention cycles between auditory and visual stimuli under conditions of high short-term demand, and that the cycles were of irregular intensity and duration across individuals. More finely grained timeseries analyses would be needed to determine a more specific non-linear pattern if one exists. 


\subsection{Limitations and future research}

Several features of the present study could be changed in future research to enhance the generalisability of the cross-modal interference phenomenon in larger perception-action sequences. Within the $\mathrm{N}$-back type of experiment, the visual component could be changed so that single target images appear in different spatial locations on the screen; this manipulation would inform whether cross-modal inhibition extends to spatial perceptions as well as shape perception. Similarly, the audio stimuli could be changed from spoken letters of the alphabet to tones or tone patterns.

Different types of tasks could be studied that emulate other types of real-world tasks. $\mathrm{N}$-back tasks, for instance, would be representative of vigilance or security functions where the operator needs to recognise people or events that crossed the visual screen in the recent past. Once can anticipate, however, that there might be a trade-off between the realism or ecological validity of an experimental task and the ability to discern clear-cut examples of an isolated cognitive process. Perception-action schemata can vary in complexity, and the complexity could have some impact on the cross-modal interference at the start of each cycle. Cross-modal blink has only been studied experimentally with simplistic perception-action requirements; studies that were framed around attentional cycles have imposed deeper perception--action requirements on the participants (e.g. Ye et al. 2009).

Finally, there is the role of cross-modal interference in pragmatic ergonomic phenomena. In Mkrtchyan et al.'s (2012) experimental interface, one would have thought that the rule of redundancy improving reliability would apply to the introduction of an auditory alert to a primarily visual task. The opposite happened instead, and future research should explore the extent to which cross-modal interference is equally likely for conjoint and disjointed stimuli (the latter were used in the present experiment) and where the rule of redundancy improving reliability continues to hold. It was not clear why Mkrtchyan et al. attempted to induce cycling between the task and distractions, but their finding that the attempt only worked on the most distracted participant is informative and suggests that further conflicts between on-task functions and distractions should be explored further from the framework of perceptual cycle theory. Their study and the present one also raise another issue, which is that the use of rigid periodicity in the presentation of stimuli in experiments may be at odds with the temporal presentation of real-world affordances.

\subsection{Theoretical implications}

Cross-modal blink presented a boundary condition for resource theory (Wickens 2002, 2008) and thus a potential problem in ergonomics (Theeuwes et al. 2007). The typical task in a cross-modal blink experiment presented stimuli in different modes and required minimum cognitive processing. Thus, if bottlenecks were unlikely because of the different stimulus modes, the blink phenomenon presented a contradiction.

The present study added on a challenging memory demand that was applied to both auditory and visual channels. The resources required at the earliest stage of processing were also different in the sense that one was language-based and the other was pictorial recognition. The subsequent processing schema that was applied to both channels consisted of four basic steps: hold stimulus in memory, update the short list in memory, match, and respond. By applying the same schema to two different channels, the $\mathrm{N}$-back task did present an opportunity for task switching, which would imply that at 
least one other schema was in play. We now know from recent studies (Guastello, Gorin et al. 2012, 2013) that there is substantial variability in the task-switching schemata that people could adopt, and that their task-switching schemata are often sub-optimal. The memory schemata do not by themselves present any additional boundary conditions to resource theory. Rather, the important point is that the bias against visual processing persisted beyond the initial perception phase of the cognitive process.

The conditions covered by resource theory, nevertheless, aptly explain the events occurring subsequently to the bottleneck circuit where the mainstay of the more complex work activities is actually taking place. It also appears to be consistent with the $81 \%$ of observations (based on Table 1) where interference did not occur. Resource theory was probably never intended to apply to responses to multiple stimuli occurring within the first $300 \mathrm{msec}$ associated with the IRP. The events occurring between 0.5 and $3.0 \mathrm{sec}$ or a 3-min span of 60 trials are another matter, and thus the target of the present investigation. Three min could be a critical interval in urgent vigilance tasks or other humancomputer interactions.

Although resource theory and the working explanation of attentional blink are both primarily centred on load issues, fatigue could be responsible for lowering load capacity. Fatigue could also arise from overworking the circuit that is responsible for bottlenecks when they occur. The dynamics of fatigue and workload in the $\mathrm{N}$-back task were investigated further in another study (Guastello et al., in press). It now appears that several individual differences in cognitive flexibility are responsible in part for changes in performance when the workload shifted from 2-back to 3-back. Other individual differences in fluid intelligence partly explained the patterns of learning the task, susceptibility to fatigue and the phase shift associated with recovery from fatigue. The phase shift that seemed to have occurred after block 9 was similar to temporal patterns that were reported earlier (Guastello, Boeh et al. 2013) and interpreted as reorganisations of available degrees of freedom in cognitive or neural processes (Guastello, Boeh, Shumaker and Schimmels 2012). On the other hand, the actual cognitive degrees of freedom are not directly visible in every type of experimental task, which presents a challenge for designing future experiments.

It is possible that when put into a sufficient workload or fatigue-inducing constriction, attention cycles between stimuli coming in from different sensory channels or from different sources that utilise the same channels. This much is expected from Neisser's (1976) and Gibson's (1979) contributions concerning perceptual cycling, schemata, and affordances. The greater degree of visual versus auditory impairment, in either the standard cross-modal blink or the form observed in working memory, indicates that the process is one of the forced oscillator rather than a pure oscillator. A parameter would need to be identified that controls the forcing level. Another situational difference is whether the auditory and visual stimuli are separate flows pertaining to separate tasks or whether the auditory and visual cues are interpreted simultaneously to produce a conjoint response that requires both cues to be present to map to an accurate response. The schemata would be less connected in the disjointed tasks than the conjoint task.

The concept of time ecologies was useful for supporting, or at least suggesting, the main hypothesis that cross-modal interference would be observed at broader time scales than those used in experimental designs in which cross-modal blink has been studied to date. Furthermore, there were 
positive and negative changes in performance that were observed over still longer time scales that were at least partially related to learning, fatigue, and recovery from fatigue. At the other end of the time ecology, neurological columns are rife with oscillatory dynamics that are moderately coupled and synchronised. Although there is no clear path between elementary neurological dynamics and cycles in the use of schemata, it would be likely that one could emerge from the other, and further studies would be warranted on this point.

\section{Acknowledgements}

The authors wish to thank Mitchell Nyfeller, Megan Fabisch, Michaela Krumholz, Paul Timm, and James Shaline for their assistance with data collection.

\section{References}

Ackerman, P. L. (Ed.). 2011. Cognitive Fatigue. Washington, DC: American Psychological Association. Ahmed, S., K. Babski-Reeves, J. DuBien, and H. Webb. 2014. "A Proposed Relationship Between Time and Load to Quantify Fatigue." Proceedings of the Human Factors and Ergonomics Society 58: 1556-1560.

Akurek, E. G., S. A. H. Eshuis, M. R. Nieuwenstein, J. D. Saija, D. Baskent, and B. Hommel. 2012. "Temporal Target Integration Underlies Performance at Lag 1 in the Attentional Blink." Journal of Experimental Psychology: Human Perception and Performance 38: 1448-1464.

Baddeley, A. 2003. "Working Memory: Looking Back and Looking Forward." Nature Reviews Neuroscience 4: 829-839.

Baird, B. 1999. "An Oscillating Cortical Model of Auditory Attention and Electrophysiology." Neurocomputing 26-27: 319-328.

Batterink, L., C. M. Karns, and H. Neville. 2012. "Dissociable Mechanisms Supporting Awareness: The P300 and Gamma in a Linguistic Attentional Blink." Cerebral Cortex 22: 2733-2744.

Bills, A. G. 1931. Blocking: A New Principle in Mental Fatigue." American Journal of Psychology 67: 230245.

Bressler, S. L., and W. J. Freeman. 1980. "Frequency Analysis of Olfactory System EEG in the Cat, Rabbit, and Rat." Electroencephalography: Clinical Neurophysiology 50: 19-24.

deJong, P. J., E. H. Koster, R. van Wees, and S. Martens. 2009. "Emotional Facial Expressions and the Attentional Blink: Attenuated Blink for Angry and Happy Faces." Cognition and Emotion 23: 1640-1652.

Dux, P. E., and R. Marios. 2009. "The Attentional Blink: A Review of Data and Theory." Attention, Perception, and Psychophysics 71: 1683-1700.

Friston, K. 2010. "The Free-Energy Principle: A Unified Brain Theory?" Nature Reviews: Neuroscience 11 (2): 127-138.

Gibson, J. J. 1979. The Ecological Approach to Visual Perception. Boston, MA: Houghton Mifflin.

Gregson, R. A. M. 1992. n-Dimensional Nonlinear Psychophysics. Hillsdale, NJ: Lawrence Erlbaum Associates.

Guastello, S. J. 2003. "Nonlinear Dynamics, Complex Systems, and Occupational Accidents." Human Factors in Manufacturing 13: 293-304.

Guastello, S. J. 2006. Human Factors Engineering and Ergonomics: A Systems Approach. Mahwah, NJ: Lawrence Erlbaum Associates.

Guastello, S. J. 2010. “Nonlinear Dynamics of Team Performance and Adaptability in Emergency Response." Human Factors 52: 162-172. 
Guastello, S. J. 2014. Human Factors Engineering and Ergonomics: A Systems Approach. 2nd ed. Boca Raton, FL: CRC Press.

Guastello, S. J., H. Boeh, H. Gorin, S. Huschen, N. E. Peters, M. Fabisch, and K. Poston. 2013. “Cusp Catastrophe Models for Cognitive Workload and Fatigue: A Comparison of Seven Task Types." Nonlinear Dynamics, Psychology, and Life Sciences 17: 23-47.

Guastello, S. J., H. Boeh, M. Schimmels, H. Gorin, S. Huschen, E. Davis, N. E. Peters, M. Fabisch, and K. Poston. 2012. "Cusp Catastrophe Models for Cognitive Workload and Fatigue in a Verbally-Cued Pictorial Memory Task." Human Factors 54: 811-825.

Guastello, S. J., H. Boeh, C. Shumaker, and M. Schimmels. 2012. "Catastrophe Models for Cognitive Workload and Fatigue." Theoretical Issues in Ergonomics Science 13: 586-602.

Guastello, S. J., H. Gorin, S. Huschen, N. E. Peters, M. Fabisch, and K. Poston. 2012. "New Paradigm for Task Switching Strategies While Performing Multiple Tasks: Entropy and Symbolic Dynamics Analysis of Voluntary Patterns." Nonlinear Dynamics, Psychology, and Life Sciences 16: 471497.

Guastello, S. J., H. Gorin S. Huschen, N. E. Peters, M. Fabisch, K. Poston, and K. Weinberger. 2013. "The Minimum Entropy Principle and Task Performance." Nonlinear Dynamics, Psychology, and Life Sciences 17: 405-424.

Guastello, S. J., M. Malon, P. Timm, K. Weinberger, H. Gorin, M. Fabisch, and K. Poston. 2014. "Catastrophe Models for Cognitive Workload and Fatigue in a Vigilance Dual-Task." Human Factors 56: 737-751.

Guastello, S. J., and D. W. McGee. 1987. "Mathematical Modeling of Fatigue in Physically Demanding Jobs." Journal of Mathematical Psychology 31: 248-269.

Guastello, S. J., A. F. Peressini, and R. W. Bond, Jr. 2011. "Orbital Decomposition for III-Behaved EventSequences: Transients and Superordinate Structures." Nonlinear Dynamics, Psychology, and Life Sciences 15: 465-476.

Guastello, S. J., K. Reiter, M. Malon, P. Timm, and J. Shaline. In press. "Catastrophe Models for Cognitive Workload and Fatigue in N-back Tasks." Nonlinear Dynamics, Psychology, and Life Sciences.

Guastello, S. J., K. Reiter, A. Shircel, P. Timm, M. Malon, and M. Fabisch. 2014. "The PerformanceVariability Paradox, Financial Decision Making, and the Curious Case of Negative Hurst Exponents." Nonlinear Dynamics, Psychology, and Life Sciences 18: 297-328.

Guastello, S. J., A. Shircel, M. Malon, and P. Timm. 2015. "Individual Differences in the Experience of Cognitive Workload." Theoretical Issues in Ergonomics Science. 16: 20-52.

Guastello, S. J., A. Shircel, D. Poggi, M. Malon, P. Timm, K. Weinberger, K. Reiter, and M. Fabisch. 2013. "Cusp Catastrophe Models for Cognitive Workload and Fatigue: A New Paradigm for Studying Biases in Financial Decision Making." Paper presented at the 23rd Annual International Conference of the Society for Chaos Theory in Psychology and Life Sciences, Portland, OR, July 25-27.

Hancock, P. A. 2013. "In Search of Vigilance: The Problem of latrogenically Created Psychological Phenomena." American Psychologist 68: 97-109.

Haroush, K., L. Y. Deouell, and S. Hochstein. 2011. "Hearing While Blinking: Multisensory Attentional Blink Revisited." The Journal of Neuroscience 31: 922-927.

Hong, S. L. 2010. "The Entropy Conservation Principle: Applications in Ergonomics and Human Factors." Nonlinear Dynamics, Psychology, and Life Sciences 14: 291-315.

Hoskinson, P., and J. Toomin. 2012. "Brain Workshop." Accessed May 17, 2013. http://brainworkshop.sourceforge.net. 
Johnston, J. C., R. McCann, and R. Remington. 1995. “Chronometric Evidence for Two Types of Attention." Psychological Science 6: 365-369.

Kane, M. J., and R. W. Engle. 2002. "The Role of Prefrontal Cortex in Working-Memory Capacity, Executive Attention, and General Fluid Intelligence: An Individual-Differences Perspective." Psychonomic Bulletin and Review 9: 617-671.

Kantowitz, B. H. 1985. "Channels and Stages in Human Information Processing: A Limited Analysis of Theory and Methodology." Journal of Mathematical Psychology 29: 135-174.

Kelly, A. J., and P. E. Dux. 2011. "Different Attentional Blink Tasks Reflect Distinct Information Processing Limitations: An Individual Differences Approach." Journal of Experimental Psychology: Human Perception and Performance 37: 1867-1873.

Klein, G. 1989. "Recognition-Primed Decisions." Advances in Man-Machine Systems Research 5: 4792.

Koehler, G. 2003. "Time, Complex Systems, and Public Policy: A Theoretical Foundation for Adaptive Policy Making." Nonlinear Dynamics, Psychology, and Life Sciences 7: 99-114.

Logie, R. H. 2011. "The Functional Organization and Capacity Limits of Working Memory." Current Directions in Psychological Science 20: 240-245.

MacLean, M. H., and K. M. Arnell. 2010. "Personality Predicts Temporal Attention Costs in the Attentional Blink Paradigm." Psychonomic Bulletin and Review 17: 556-562.

Mandelbrot, B. B. 1997. Fractals and Scaling in Finance: Discontinuity, Concentration and Risk. New York: Springer.

Marti, S., M. Sigman, and S. Dehaene. 2012. "A Shared Cortical Bottleneck Underlying Attentional Blink and Psychological Refractory Period." Neurolmage 59: 2883-2898.

Mkrtchyan, A. A., J. C. Macbeth, E. T. Solovey, J. C. Ryan, and M. L. Cummings. 2012. "Using VariableRate Alerting to Counter Boredom in Human Supervisory Control." Proceedings of the Human Factors and Ergonomics Society 56: 1441-1445.

Musch, K., A. K. Engel, and T. R. Schneider 2012. "On the Blink: The Importance of Target-Distractor Similarity in Eliciting an Attentional Blink with Faces." PLoS One 7 (7): e41257.

Neisser, U. 1976. Cognition and Reality: Principles and Implications of Cognitive Psychology. San Francisco, CA: W. H. Freeman.

Ott, E., C. Grebogi, and J. A. Yorke. 1990. “Controlling Chaos.” Physical Review Letters 64: 1196-1199.

Pashler, H., and J. C. Johnston. 1998. "Attention Limitations in Dual-Task Performance." In Attention, edited by H. Pashler, 155-189. East Sussex: Psychology Press.

Plant, K. L., and N. A. Stanton. 2012. "Why Did the Pilots Shut Down the Wrong Engine? Explaining Errors in Context Using Schema Theory and the Perceptual Cycle Model." Safety Science 30: 300-315.

Raymond, J. E., K. L. Shapiro, and K. M. Arnell. 1992. "Temporary Suppression of Visual Processing in an RSVP Task: An Attentional Blink?" Journal of Experimental Psychology: Human Perception, and Performance 18: 849-860.

Schadow, J., D. Lenz, S. Thaerig, N. A. Busch, I. Frund, J. W. Rieger, and C. S. Herrmann. 2007. "Stimulus Intensity Affects Early Sensory Processing: Visual Contrast Modulates Evoked Gamma-Band Activity in Human EEG." International Journal of Psychophysiology 66: 28-36.

Spivey, M. J., and R. Dale. 2006. "Continuous Dynamics in Real-Time Cognition." Current Directions in Psychological Science 15: 207-211.

Stanton, N. A., P. R. Chambers G., and J. Piggot. 2001. "Situational Awareness and Safety." Safety Science 39: 189-204. 
Theeuves, J., E. van der burg, C. N. L. Olivers, and A. Bronkhorst. 2007. "Cross-Modal Interaction Between Sensory Modalities: Implications for the Design of Multisensory Displays." In Attention: From Theory to Practice, edited by A. F. Kramer, D. A. Wiegman, and A. Kirklik, 196-205. New York: Oxford University Press.

Van Dam, N. T., M. Earleywine, and J. Alterriba. 2012. "Anxiety Attenuates Awareness of Emotional Faces During Rapid Serial Visual Presentation." Emotion 12: 796-806. Ward, L. M. 2004. "Oscillations and Synchrony in Cognition." In Coordination Dynamics: Issues and Trends, edited by V. K. Jirsa, and J. A. S. Kelso, 217-242. New York: Springer.

Welford, A. T. 1952. "The 'Psychological Refractory Period' and the Timing of High Speed Performance: A Review and a Theory." British Journal of Psychology 43: 2-19.

Welford, A. T. 1980. "The Single-Channel Hypothesis." In Reaction Time, edited by A. T. Welford, 215252. New York: Academic Press.

Whittle, P. 2010. Neural Nets and Chaotic Carriers. 2nd ed. London: Imperial College Press.

Wickens, C. D. 2002. "Multiple Resources and Performance Prediction." Theoretical Issues in Ergonomics Science 3: 159-177.

Wickens, C. D. 2008. "Multiple Resources and Mental Workload." Human Factors 50: 449-455.

Wynna, J. K., B. Breitmeyer, K. H. Nuechterlein, M. F. Green. 2006. "Exploring the Short Term Visual Store in Schizophrenia Using the Attentional Blink." Journal of Psychiatric Research 40: 599605.

Ye, L., W. Carwell, and L. S. Mark. 2009. "Perceiving Multiple Affordances for Objects." Ecological Psychology 21: 185-217.

Zaheer, S., S. Albert, and A. Zaheer. 1999. "Time Scales and Organizational Theory." Academy of Management Review 24: 725-741. 